\title{
An acute care collaboration vanguard: translating a perioperative service from a tertiary referral centre to a district general hospital (DGH)
}

\author{
Authors: Ruth De Las Casas, ${ }^{A}$ Anna Whittle, ${ }^{B}$ Catherine Meilak, ${ }^{B}$ Judith Partridge ${ }^{A}$ and Jugdeep Dhesi ${ }^{A}$
}

\begin{abstract}
Aims
To translate the Proactive care of Older People undergoing Surgery service (POPS) from Guy's and St Thomas' NHS Foundation Trust (GSTT) to Dartford and Gravesham Foundation Trust (DGT). POPS at GSTT has demonstrated geriatrician-led perioperative care improves outcomes and reduces length of stay. Vanguard status between GSTT and DGT facilitated scoping work which demonstrated a local vascular POPS service at DGT would improve user-experience and service accessibility. It was hypothesised that implementing POPS would improve patient, clinician, and processreported outcomes at DGT.
\end{abstract}

\section{Methods}

A local POPS vascular clinic was introduced at DGT in October 2016.

The adoption of a novel cross-site collaboration model between GSTT and DGT supported data collection, service planning and staff development. Additionally, it maintained links for governance, educational and academic activities without acquisition or merger.

Service-user engagement is key to POPS success, and there has been continuous effort to ensure co-production with events such as patient coffee mornings.

Multidisciplinary learning is central to POPS, and an education programme in perioperative medicine, taught by surgeons, geriatricians and anaesthetists has been implemented at DGT.

The challenge of translating an existing service into a different context is novel. Process-mapping, active stakeholder engagement, and collection of service-user feedback has supported considerate integration at DGT.

\section{Results}

Following the successful initiation of the vascular clinic, there was interest in expanding POPS into other surgical specialties. To quantify need for the service, data within general surgery was collected, and demonstrated a predominantly old and comorbid surgical inpatient population. A questionnaire found 19 of 20 surgeons supported closer integration of geriatricians in care of older surgical patients.

Substantive funding to continue the POPS vascular service and to expand into general surgery was secured in July 2017. Funding for a Darzi Fellow was granted to evaluate the translation, and apply learning to the field of perioperative medicine and improvement science.

At DGT the POPS service falls under the Surgical Directorate. This unprecedented arrangement aims to optimise partnership and learning between the POPS team, surgeons, anaesthetists and intensivists.

\section{Conclusion}

The translation of a large and established teaching hospital service into a new service within a DGH, is pioneering and continues to demand a problem-solving approach. Placement within the Surgical Directorate, and commitment to cross-site collaboration with GSTT are both innovative but instrumental approaches to the service design, which will improve care of this vulnerable patient group, as well as supporting training, research, and quality improvement within both DGT and the developing field of perioperative medicine. 